\title{
Determinação da Dose dos Fótons Contaminantes de Feixes de Elétrons Clínicos usando o Método de Recozimento Simulado
} Generalizado

\author{
Determination of Photon Contamination Dose of Clinical Electron Beams \\ using the Generalized Simulated Annealing Method
}

\author{
Jorge H. Wilches Visbal ${ }^{1}$, Alessandro M. Da Costa ${ }^{2}$ \\ ${ }_{1,2}$ Faculdade de Filosofia, Ciências e Letras de Ribeirão Preto (USP), Ribeirão Preto (SP), Brasil
}

\section{Resumo}

Os feixes de elétrons clínicos são compostos por uma mistura de elétrons puros e fótons de freamento produzidos nas estruturas internas do cabeçote do acelerador bem como no ar. O conhecimento acurado desses componentes é importante para o cálculo da dose quanto para o planejamento do tratamento. Existem ao menos duas abordagens para determinar a contribuição dos fótons na porcentagem de dose em profundidade (PDP) dos elétrons clínicos: a) Método Analítico que calcula a dose dos fótons a partir da prévia determinação do espectro dos fótons de freamento incidentes; b) Método de Ajuste baseado em uma formula biexponencial semiempírica em que quatro parâmetros devem ser estabelecidos a partir de métodos de otimização. Os resultados revelam que o método de recozimento simulado generalizado consegue calcular a dose dos fótons contaminantes superestimando a dose na cauda não mais do que $0,6 \%$ da dose máxima (elétrons e fótons).

Palavras-chave: recozimento simulado generalizado; fótons contaminantes; elétrons clínicos; radioterapia; PDP; MATLAB; otimização.

\begin{abstract}
Clinical electron beams are composed of a mixture of pure electrons and bremsstrahlung photons produced in the structures of the accelerator head as well as in the air. Accurate knowledge of these components is important for calculating the dose and for treatment planning. There are at least two approaches to determine the contribution of the photons in the percentage depth dose of clinical electrons: a) Analytical Method that calculates the dose of the photons from the previous determination of the spectrum of the incident bremsstrahlung photons; $b$ ) Adjustment method based on a semi-empirical biexponential formula where four parameters must be established from optimization methods. The results show that the generalized simulated annealing method can calculate the photon contamination dose by overestimating the dose in the tail no more than $0.6 \%$ of the maximum dose (electrons and photons).
\end{abstract}

Keywords: generalized simulated annealing; clinical electrons; radiation therapy; PDD; MATLAB; optimization.

\section{Introdução}

Os feixes de elétrons são frequentemente usados no tratamento de tumores superficiais ${ }^{1}$ toda vez que se caracterizam por entregar uma dose relativamente uniforme ao longo do volume alvo que logo em seguida cai rapidamente até se estabilizar em uma dose de baixo nível chamada de cauda de bremsstrahlung.

A contribuição dos fótons de bremsstrahlung da cauda que aparece na parte final da porcentagem de dose em profundidade dos feixes de elétrons deve ser conhecida a fim providenciar mecanismos que poupem a dose desnecessária nos órgãos embaixo do volume alvo, sobretudo com o aumento da energia nominal.

A terapia com elétrons vem-se utilizando do início da década de 1950, mas só os aceleradores lineares modernos conseguiram fornecer várias energias de elétrons na faixa dos 4-25 MeV1.

Os tamanhos de campos usados na radioterapia com elétrons são tipicamente pequenos ${ }^{2}$ sendo moldados com lâminas de chumbo ou blocos de cerrobend.
O planejamento de um tratamento com feixe de elétrons requer cálculos acurados da distribuição e do perfil de dose para todos os tamanhos de campo.

Feixes clínicos de elétrons contêm uma mistura de fótons de freamento e elétrons puros.

A contribuição dos fótons de freamento aparece como uma cauda que diminui lentamente com a profundidade na curva de porcentagem de dose em profundidade de elétrons clínicos, além do alcance máximo dos elétrons ${ }^{3}$.

Há dois tipos de contribuições à dose dos fótons de freamento em um feixe clinico de elétrons ${ }^{3,4}$. Uma que provem dos fótons gerados no cabeçote do tratamento quando os elétrons interagem com os dispositivos espalhadores e definidores do feixe, chamada dose dos fótons contaminantes. A outra é produzida no interior do objeto simulador quando os elétrons interagem com as moléculas de água.

Em máquinas com elementos espalhadores, como os aceleradores lineares, a contribuição dos fótons contaminantes é significativamente maior àquela dos fótons do objeto simulador ${ }^{3,4}$.

Em geral, a dose dos fótons de freamento depende proporcionalmente da energia do feixe, do 
número atômico dos elementos degradantes tanto no acelerador quanto no objeto simulador, da espessura relativa ao alvo de tungstênio, o design do cabeçote etc ${ }^{3,4,5}$.

As curvas de distribuição de dose dos fótons contaminantes e as dos fótons gerados no objeto simulador são diferentes entre si a respeito da curva de distribuição de dose dos elétrons.

Adicionalmente, a distribuição de dose dos fótons contaminantes em feixes de elétrons clínicos possui uma forma similar à distribuição de fótons de raios$x$ de alta energia, com caraterísticas tais como uma região de build-up próxima da superfície, uma profundidade de máxima dose deslocada em profundidade seguida por uma região de queda exponencial $^{3,4,5}$.

Fica claro que o conhecimento das componentes dos fótons de freamento, sobretudo a dose dos fótons contaminantes, é relevante para o modelamento dos feixes de elétrons clínicos no planejamento do tratamento ${ }^{6}$, toda vez que se garante uma dosimetria acurada do feixe de elétrons ${ }^{5}$.

Nesse estudo, nós mostramos como o método de recozimento simulado generalizado é capaz de resolver o modelo semiempírico para a dose dos fótons contaminantes de maneira rápida e efetiva.

\subsection{Modelamento do Problema}

Existem ao menos duas formas de calcular a dose dos fótons em um feixe de elétrons:

1. Método de extrapolação linear da cauda de fótons de freamento na PDP de elétrons clínicos ${ }^{4-8}$ pelo ajuste da equação (1)

2. Método analítico da fórmula Schiff para a determinação do espectro de fótons de freamento e consequentemente a dose por eles depositada ${ }^{8-9}$.

Embora Faddegon et $a^{\beta}$ descreva essa última abordagem como sendo a melhor dentre as duas, nós consideramos que a necessidade de conhecer a priori algumas caraterísticas do cabeçote do tratamento ${ }^{9}$ tais como a espessura equivalente à interação do feixe com as múltiplas estruturas, a quantidade de matérias envolvidos, dentre outros, o faz menos atrativo.

A distribuição de dose dos fótons no feixe de elétrons segue uma forma similar à distribuição dos fótons de alta energia a qual, por sua vez, pode ser descrita pela seguinte equação semiempírica ${ }^{4-7}$ :

$$
D(z)=D_{c}\left(e^{-u_{p} z}-v e^{-u_{e} z}\right)
$$

Em que $D_{c}$ é uma constante de normalização, $u_{p}$ o coeficiente de atenuação dos fótons primários, $u_{e} 0$ coeficiente de atenuação dos elétrons secundários, $(1-v)$ um fator relacionado com a dose superficial e $z$ o vetor profundidade.

Como discutido por Sorcini et a/ ${ }^{5}$ e implementado por Deng et $a^{4}$ e Zhu et $a^{6}$ o processo físico de modelamento do feixe inclui a contribuição dos fótons do objeto simulador no kernel dos elétrons, entretanto usa-se um kernel separado para os fótons contaminantes.
Daqui que o interesse sempre será a determinação da contribuição dos fótons gerados no cabeçote pois são estes os que atingem a superfície do objeto simulador de água e durante a reconstrução inversa do espectro de elétrons eles não podem ser extraídos da PDP clinica ${ }^{10}$.

Assim sendo, nosso intuito será a determinação dos quatro parâmetros na equação (1) tal que com eles possamos descrever apropriadamente a porção da dose dos fótons gerados no cabeçote nas curvas de PDP de elétrons clínicos no eixo central.

Para tal finalidade, nós fizemos uso do método de recozimento simulado generalizado, uns dos métodos de otimização estocástica mais reconhecidos da computação científica, o qual explicaremos brevemente na seção a seguir.

\subsection{Método de Recozimento Simulado Generali- zado}

Recozimento simulado é um método metaheurístico que resolve problemas multidimensionais de otimização global com alta eficiência. Ele atraiu atenção significativamente devido à sua adequação para problemas de larga escala, especialmente em aqueles em que o mínimo global desejado acha-se escondido entre muitos mínimos locais ${ }^{10.11}$.

O método de recozimento simulado é baseado no processo termodinâmico pelo qual um metal atinge uma estrutura cristalográfica livre de defeitos e imperfeições. Basicamente, o metal é aquecido a uma temperatura muito alta (muitos estados permitidos) e logo após ele é arrefecido a uma temperatura muito baixa (apenas um estado permitido). Se o processo de resfriamento for levado a cabo com bastante lentidão, os átomos formarão uma estrutura estável ou mínimo global de energia. Em contraste, se o processo de resfriamento for rápido alcançarase um estado com muitos defeitos e irregularidades na estrutura metálica ou estado metaestável correspondente a um mínimo local de energia ${ }^{10}$.

Ao contrário dos métodos de otimização determinística que convergem rapidamente para soluções associadas a mínimos locais, o recozimento simulado pode pular esses mínimos locais e imediatamente atingir o mínimo global $^{12}$

O método de Recozimento Simulado Generalizado (GSA: Generalized Simulated Annealing) foi apresentado por Tsallis \& Stariolo ${ }^{13}$ em 1996 e baseia-se na estatística não-extensiva de Tsallis $^{14}$. $O$ método é uma generalização do método de recozimento simulado clássico (CSA: Classical Simulated Annealing $)^{15}$ e o de recozimento simulado rápido (FSA: Fast Simulated Annealing) ${ }^{16}$ sendo mais rápido e eficiente que estes ${ }^{17}$.

$O$ recozimento simulado generalizado do Tsallis (GSA) consiste de três etapas ${ }^{13}$ :

- A função de distribuição de probabilidade ou a distribuição de visitação, $g_{q_{v}}\left(\Delta x_{t}\right)$;

- A probabilidade de aceitação $P_{q_{a}}\left(x_{t} \rightarrow x_{t}+\right.$ 1);

- A programação de resfriamento ou redução de temperatura $T_{q_{v}}$. 
O primeiro passo é a amostragem de Tsallis ${ }^{13} \mathrm{com}$ base em uma distorção da distribuição de visitação de Cauchy-Lorentz,

$$
\begin{array}{r}
g_{q_{v}}\left(\Delta x_{t}\right)=\left(\frac{q_{v}-1}{\pi}\right)^{\frac{D}{2}} \frac{\Gamma\left(\frac{1}{q_{v}-1}+\frac{D-1}{2}\right)}{\Gamma\left(\frac{1}{q_{v}-1}+\frac{1}{2}\right)} \\
. \frac{T_{q_{v}}-\frac{D}{\left(3-q_{v}\right)}}{1+\left(q_{v}-1\right) \frac{\left(\Delta x_{t}\right)^{2}}{\frac{2}{T_{q_{v}}^{\left(3-q_{v}\right)}}}}
\end{array}
$$

Com dimensão de busca $D$, atualização de estado $\Delta x_{t}=x_{t+1}-x_{t}$ e parâmetro de visitação $q_{v}$ que controla a forma e abertura da distribuição.

O segundo passo é a aceitação dos estados. Se a energia do novo estado for menor do que a energia do estado antigo, o novo estado será aceito, caso contrário, o novo estado será aceito aleatoriamente. A última propriedade é importante para evitar ficar preso em estados metaestáveis. Um algoritmo Metrópoles generalizado é usado para a probabilidade de aceitação ${ }^{12}$,

$$
\begin{aligned}
& P_{q_{a}}\left(x_{t} \rightarrow x_{t}+1\right) \\
& = \begin{cases}0 & \Delta E_{t}<0 \\
\left(1-\left(1-q_{a}\right) \frac{\Delta E_{t}}{k T_{q_{a}}}\right)^{1 /\left(1-q_{a}\right)} & \Delta E_{t} \geq 0\end{cases}
\end{aligned}
$$

Com temperatura de aceitação $T_{q_{a}}$, constante de Boltzmann $k$ e parâmetro de aceitação $1<q_{a}<3$.

Um esquema simples de temperatura de aceitação para acelerar a convergência é obtido dividindo a temperatura de visitação pelo número de etapas de tempo ${ }^{12}$, ou seja, $T_{q_{a}}=T_{q_{v}} / t$.

O último passo é o cronograma de resfriamento ou redução de temperatura de visitação,

$$
T_{q_{v}}(t)=T_{q_{v}}(1) \frac{2^{q_{v}-1}-1}{(1+t)^{q_{v}-1}-1}
$$

Perceba que se $q_{v}=1$ e $q_{a}=1$, GSA recupera CSA (máquina de Boltzmann) e quando $q_{v}=2 \mathrm{e}$ $q_{a}=1$, GSA recupera FSA (máquina de Cauchy). Tsallis \& Stariolo ${ }^{13}$ reportaram que o esquema com maior eficiência é aquele em que $q_{v}=2.7$ e $q_{a}$ tão negativo quanto possível, por exemplo, $q_{a}=-5.0$.

Indubitavelmente, a geração de números aleatórios que obedecem à distribuição visitante Tsallis dada pela equação (2) é a parte mais complexa do método de recozimento simulado generalizado.

Muito embora dessa complexidade, em 2006, Schanze ${ }^{18}$ conseguiu resolver o problema desenvolvendo um gerador exato de números aleatórios de Tsallis de dimensão $D$ conforme necessário para a amostragem do espaço de estados de GSA de dimensão $D$, demonstrando que a variável aleatória de Tsallis é uma multivariada t ou que segue uma distribuição t-student.

\section{Materiais e Métodos}

Para a obtenção dos resultados o algoritmo de recozimento simulado generalizado foi programado no Matlab sob uma função nomeada GSA.

Seguidamente determina-se a dose da cauda extrapolando linearmente os valores da PDP de elétrons desde o alcance máximo dos elétrons $(R p+$ $2 \mathrm{~cm}$ ) até o alcance prático e daí até a profundidade de dose superficial ${ }^{4}$.

Dado que na dose da cauda estão contidas às duas contribuições e o intuito é determinar só a dos fótons contaminantes, subtrai-se a contribuição dos fótons do objeto simulador antes da minimização.

A dose dos fótons do objeto simulador é apontada como sendo uma porcentagem da dose dos fótons totais (dose da cauda) ${ }^{3}$.

Esse vetor, dose da cauda, será aquele do qual deverão ser extraídos os valores da equação (1) por meio da minimização da normal residual a seguir:

$$
\min \left\{\left\|D_{c}\left(e^{-u_{p} z}-v e^{-u_{e} z}\right)-D_{\text {cauda }}\right\|^{2}\right\}
$$

Apenas três dos quatro parâmetros serão determinados, o $u_{p}$ vem dado em função da energia média do feixe de elétrons clinicos ${ }^{6}: u_{p}=0,084 E_{\text {med }}^{-0,218} \mathrm{em}$ que $E_{\text {med }}=2,33 R_{50}$.

Além do mais, estabeleceram-se restrições nos quatro parâmetros conforme os valores mínimos e máximos reportados por Brahme \& Svensson ${ }^{7}$. Para a profundidade de dose máxima $d_{\text {máx }}=0,145 E_{\text {med }}$ permitiram-se incertezas de $\pm 2 \mathrm{~mm}$. As tentativas iniciais de solução estipularam-se em zero.

Finalmente, seguiu-se o seguinte procedimento iterativo:

- Executa-se o código com GSA enquanto as restrições para os três parâmetros não forem satisfeitas.

- $\quad$ Cada solução que satisfizer às restrições é armazenada em um ciclo externo até completar 50 soluções.

- Se faz a média das 50 soluções para obter os três valores finais dos parâmetros e o vetor dose dos fótons contaminantes.

O software utilizado na programação e consecução de resultados: Matlab R2015a, plataforma Windows 7, SP1. CPU: 2,3GHz e RAM: 4Gb.

\section{Resultados}

A dose dos fótons contaminantes para cada um dos feixes de elétrons de energia nominal 6, 9, 12 e $15 \mathrm{MeV}$ é mostrada na figura 1.

As curvas da dose dos fótons contaminantes apresentam-se em forma crescente tal que a curva de cor azul representa o feixe de elétrons com a menor dose média e a de cor roxo o feixe de elétrons com a maior dose média, como mostrado na figura 1. 


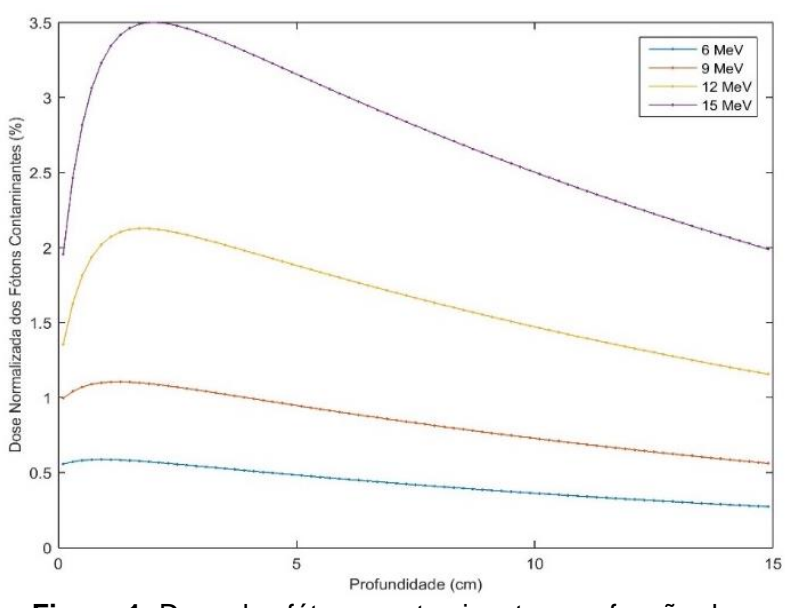

Figura 1. Dose dos fótons contaminantes em função da profundidade, normalizada à dose total do feixe de elétrons para um tamanho de campo de $10 \times 10 \mathrm{~cm}^{2}$ e distancia fonte-superfície de $100 \mathrm{~cm}$.

Na figura 2 visualiza-se a diferença na PDP dos elétrons clinicos quando não considerando a adição da dose dos fótons contaminantes (a) e quando considerada a contribuição dos mesmos (b).
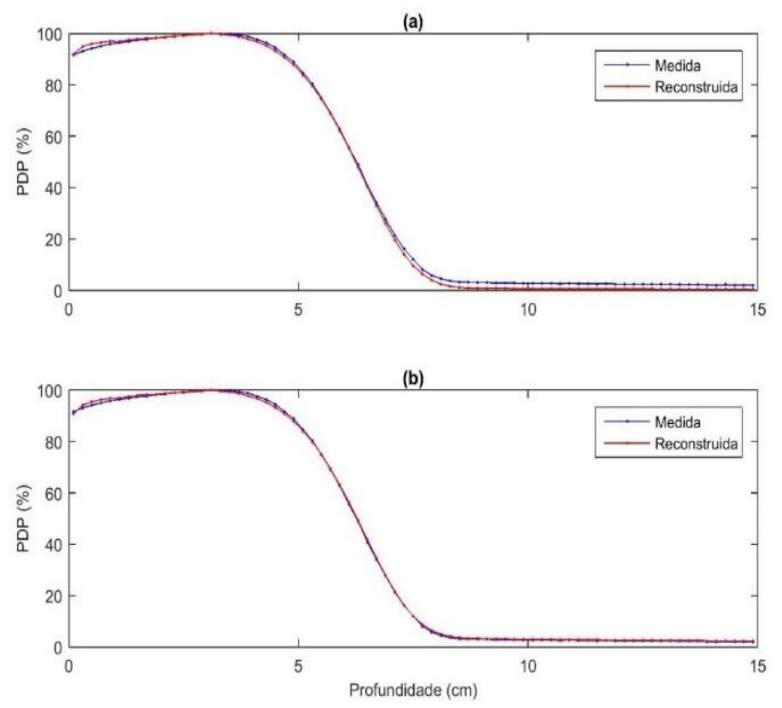

Figura 2. Comparação entre as PDP sem considerar e considerando o aporte dos fótons contaminantes para o feixe de elétrons clínicos de energia nominal de $15 \mathrm{MeV}$; (a) representa a curva de PDP sem incluir a dose dos fótons contaminantes enquanto (b) inclui a dose dos fótons contaminantes.

Para evidenciar a influência da dose dos fótons contaminantes na reconstrução do espectro dos elétrons clínicos utilizamos apenas o feixe de maior energia que aqui compete, o de $15 \mathrm{MeV}$, pois somente neste a desconsideração da dose dos contaminantes levou a desaprovação na taxa segundo o Índice Gamma $(Г)^{19}$ como visto na tabela 1.

A taxa de aprovação do índice gamma refere-se à quantidade de pontos sobre o total com Índice Gamma menor ou igual a um $(\Gamma \leq 1)$, segundo tolerâncias pré-estabelecidas na distância para concordância $(\mathrm{mm})$ e na diferença da dose (\%).

O Índice Gamma utilizado, $2 \% / 2 \mathrm{~mm}$, é o sugerido pela American Association of Physicists in Medicine $(A A P M)^{20}$. A taxa de aprovação escolhida foi do $95 \%{ }^{21}$.
Tabela 1. Erro médio absoluto e taxa de aprovação do índice gamma para os diferentes feixes. FC: fótons contaminantes.

\begin{tabular}{|c|c|c|c|c|c|}
\hline $\begin{array}{c}\text { Ener- } \\
\text { gia No- } \\
\text { minal } \\
(\mathrm{MeV})\end{array}$ & $\begin{array}{c}\text { Delta da } \\
\text { Dose na } \\
\text { Cauda } \\
(\%)\end{array}$ & \multicolumn{2}{|c|}{$\begin{array}{c}\text { Erro médio } \\
\text { absoluto } \\
(\%)\end{array}$} & \multicolumn{2}{|c|}{$\begin{array}{l}\text { Taxa Aprova- } \\
\text { ção segundo } \\
\text { o } \\
\text { gamma } \\
\text { índice }\end{array}$} \\
\cline { 2 - 6 } & Com FC & $\begin{array}{c}\text { Com } \\
\text { FC }\end{array}$ & $\begin{array}{c}\text { Sem } \\
\text { FC }\end{array}$ & $\begin{array}{c}\text { Com } \\
\text { FC }\end{array}$ & $\begin{array}{c}\text { Sem } \\
\text { FC }\end{array}$ \\
\hline 6 & 0,11 & 0,18 & 0,39 & 100 & 100 \\
\hline 9 & 0,28 & 0,27 & 0,55 & 100 & 100 \\
\hline 12 & 0,43 & 0,37 & 0,94 & 100 & 100 \\
\hline 15 & 0,59 & 0,44 & 1,36 & 100 & 80 \\
\hline
\end{tabular}

Como uma estimativa da idoneidade da reconstrução da dose dos fótons contaminantes, definiu-se o termo Delta da Dose de Cauda (\%) como aquele que representa à diferença máxima na dose dos fótons puros na cauda entre as PDP dos elétrons clínicos.

Finalmente, a função CalcGamma ${ }^{22}$ empregou-se para calcular o Índice Gamma de cada feixe.

\section{Discussão}

A curva da dose dos fótons contaminantes por ser similar à curva da dose dos fótons de raios-x de alta energia, compartilha algumas das caraterísticas destes: enquanto o valor máximo da dose, os valores da dose na profundidade e a profundidade de máxima dose aumentam com o aumento da energia nominal, a dose superficial diminui, como visto na figura 1. Estes resultados são consistentes com aqueles detalhados nas referências 4, 6 e 8 .

Muito embora da semelhança comportamental entre a curva de dose dos fótons de alta energia e a dos contaminantes, estes últimos ao serem menos penetrantes apresentam um valor da profundidade de máxima dose menor, uma região de buildup menos abrangente, um declínio mais rápido da região de queda e, certamente, uma menor contribuição à dose total para a mesma energia nominal. Tudo isto deve-se a que o coeficiente de atenuação dos fótons primários $u_{p}$ é maior que seu par dos fótons de alta energia, para a mesma energia nominal 6 .

Segundo os dados da tabela 1, desprezar a contribuição dos fótons contaminantes à medida que a energia nominal do feixe de elétrons aumenta pode acarretar erros inaceitáveis sob os parâmetros de avaliação adotados.

Resumidamente:

i. Recusar a contribuição dos fótons contaminantes reflete um aumento no erro absoluto médio para todos as energias nominais dos feixes, especialmente para maiores energias, como visto na tabela 1. O Delta da dose na cauda, para qualquer energia, não superou o $0,6 \%$ do valor máximo da PDP dos elétrons como constatado na tabela 1. Quando os contaminantes não são levados em conta, o delta da dose na cauda sobe para 2,3\%. Na figura 2 explicita-se a diferença entre às PDP, principalmente na 
cauda, quando a dose dos contaminantes não é incluída (a) e quando sim (b).

ii. Apenas para o feixe de energia nominal de $15 \mathrm{MeV}$, tem-se um erro com significância clinica visto que a taxa de aprovação ficou embaixo do $95 \%$ quando os contaminantes não foram incluídos. As regiões da PDP com maior discrepância localizaram-se perto da superfície e na cauda dos fótons de bremsstrahlung. Além, quando os contaminantes são desprezados, a PDP reconstruída desloca-se para a superfície, conduzindo a uma diferença maior no $R_{50}$. No caso de $15 \mathrm{MeV}$, passou-se de $1 \mathrm{~mm}$ para $4 \mathrm{~mm}$ de diferença entre os $R_{50}$ (resultado não mostrado).

iii. Quando a taxa de aprovação do 95\% é mantida, mas o índice gamma passa para $3 \% / 3 \mathrm{~mm}$, o $100 \%$ dos pontos passam o teste mesmo abrindo mão da contribuição dos contaminantes. Isto é uma indicativa de que a relevância clinica depende do critério assumido.

Sempre que possível recomenda-se dar importância à dose dos fótons contaminantes a fim de garantir um apropriado modelamento do feixe de elétrons a pesar de que a relevância clinica possa variar de acordo com os critérios assumidos por cada staff do serviço de radioterapia.

\section{Conclusões}

O método de Recozimento Simulado Generalizado (GSA) é capaz de reconstruir a dose dos fótons contaminantes dentro de um erro não superior ao $0,6 \%$ da dose máxima para as energias nominais aqui avaliadas.

Desconsiderar a contribuição dos fótons contaminantes pode levar à introdução sistemática de erros no modelamento da fonte, sobretudo para feixes de mais alta energia, pudendo acarretar em uma perigosa subestimação da dose entregue em órgãos sadios localizados embaixo do tumor, além do alcance prático.

Seria interessante poder extrair experimentalmente, por meio do uso de um campo magnético defletor dos elétrons, a dose dos fótons contaminantes e compará-la com a obtida neste método.

Enfatiza-se que esse método não precisa de conhecer a estrutura ou geometria do acelerador, tão somente da curva de PDP do feixe de elétrons clínicos.

\section{Agradecimentos}

Os autores agradecem o apoio da Comissão de Aperfeiçoamento de Pessoal do Nível Superior (CAPES) e o da Fundação de Amparo e Pesquisa do Estado de São Paulo (FAPESP).

\section{Referências}

1. Strydom. W, W. Parker, and M. Olivares. "Electron beams: physical and clinical aspects." Radiation oncology physics: a handbook for teachers and students. Vienna: International Atomic Energy Agency (IAEA) (2005): 273.
2. Das, Indra J., Chee W. Cheng, and Glenn A. Healey. "Optimum field size and choice of isodose lines in electron beam treatment." International Journal of Radiation Oncology* Biology* Physics 31.1 (1995): 157-163.

3. Rustgi, Surendra N., and James E. Rodgers. "Analysis of the bremsstrahlung component in 6-18 MeV electron beams." Medical physics 14.5 (1987): 884-888.

4. Deng, Jun, et al. "Derivation of electron and photon energy spectra from electron beam central axis depth dose curves." Physics in medicine and biology 46.5 (2001): 1429

5. Sorcini, B. B., S. Hyödynmaa, and A. Brahme. "The role of phantom and treatment head generated bremsstrahlung in high-energy electron beam dosimetry." Physics in medicine and biology 41.12 (1996): 2657.

6. Zhu, Timothy C., Indra J. Das, and Bengt E. Bjärngard. "Characteristics of bremsstrahlung in electron beams." Medical physics 28.7 (2001): 1352-1358.

7. Brahme, A., and H. Svensson. "Radiation beam characteristics of a $22 \mathrm{MeV}$ microtron." Acta radiologica: oncology, radiation, physics, biology 18.3 (1979): 244-272.

8. Faddegon, B. A., and I. Blevis. "Electron spectra derived from depth dose distributions." Medical physics 27.3 (2000): 514526.

9. Li, Gui, et al. "Photon energy spectrum reconstruction based on Monte Carlo and measured percentage depth dose in accurate radiotherapy." Prog Nucl Sci Technol 2 (2011): 160164.

10. Carletti, C., P. Meoli, and W. R. Cravero. "A modified simulated annealing algorithm for parameter determination for a hybrid virtual model." Physics in medicine and biology 51.16 (2006): 3941

11. Moret, Marcelo A., et al. "Stochastic molecular optimization using generalized simulated annealing." Journal of computational chemistry 19.6 (1998): 647-657.

12. Xiang, Yang, et al. "Generalized simulated annealing for global optimization: the GenSA package." R Journal 5.1 (2013).

13. Tsallis, Constantino, and Daniel A. Stariolo. "Generalized simulated annealing." Physica A: Statistical Mechanics and its Applications 233.1-2 (1996): 395-406.

14. Tsallis, Constantino. "Possible generalization of BoltzmannGibbs statistics." Journal of statistical physics 52.1 (1988): 479-487.

15. Kirkpatrick, Scott, C. Daniel Gelatt, and Mario P. Vecchi. "Optimization by simulated annealing." science 220.4598 (1983): 671-680.

16. Szu, Harold, and Ralph Hartley. "Fast simulated annealing." Physics letters A 122.3-4 (1987): 157-162.

17. Xiang, Y., and X. G. Gong. "Efficiency of generalized simulated annealing." Physical Review E 62.3 (2000): 4473.

18. Schanze, Thomas. "An exact D-dimensional Tsallis random number generator for generalized simulated annealing." Computer physics communications 175.11 (2006): 708-712.

19. Low, Daniel A., et al. A technique for the quantitative evaluation of dose distributions. Medical physics 25.5: 656-661. 1998.

20. Chetty, Indrin J., et al. "Report of the AAPM Task Group No. 105: Issues associated with clinical implementation of Monte Carlo-based photon and electron external beam treatment planning." Medical physics 34.12 (2007): 4818-4853.

21. Sumida, lori, et al. "Novel radiobiological gamma index for evaluation of 3-dimensional predicted dose distribution." International Journal of Radiation Oncology* Biology* Physics 92.4 (2015): 779-786.

22. Geurts, 2017. 1D, 2D or 3D gamma index computation in Matlab. Disponível no seguinte enlace web: https://github.com/mwgeurts/gamma.

\section{Contato:}

Jorge H. Wilches Visbal.

Departamento de Física, Bloco 5, sala 411.

Universidade de São Paulo.

Ribeirão Preto, São Paulo, Brasil.

e-mail: jhwilchev@gmail.com 